\title{
Plasma apolipoprotein E level is associated with the risk of endobronchial biopsy- induced bleeding in patients with lung cancer
}

\author{
Saibin Wang ${ }^{1 *}$, Qian Ye ${ }^{2}$ and Xiaodong $\mathrm{Lu}^{3}$
}

\begin{abstract}
Background: Factors affecting the risk of bleeding by bronchoscopic biopsy in patients with lung cancer remain unclear. The levels of plasma apolipoprotein $\mathrm{E}$ (ApoE) that may be associated with endobronchial biopsy (EBB)-induced bleeding have never been examined.

Methods: This was a retrospective study using data collected from 615 consecutive patients who had undergone EBB and been diagnosed with primary lung cancer from January 2014 through February 2018. Patients were either classified as the bleeding group $(n=214)$ or the non-bleeding group $(n=391)$ based on the bronchoscopy report. Multiple regression analysis was done to estimate the independent relationship between ApoE levels and EBB-induced bleeding, with an adjustment for potential confounders.

Results: The mean plasma ApoE concentration was higher in the non-bleeding group compared to that in the bleeding group $(P<0.05)$. However, a non-linear relationship with threshold effects was observed between plasma ApoE levels and EBB-induced bleeding in a piecewise linear regression analysis. The risk of EBB-induced bleeding decreased with ApoE concentrations from $3.5 \mathrm{mg} / \mathrm{dL}$ up to $5.9 \mathrm{mg} / \mathrm{dL}$ (adjusted odds ratio, 0.64; 95\% confidence interval, 0.43-0.94); however, the incidence of EBB-induced bleeding increased with ApoE levels above the turning point (ApoE $=5.9 \mathrm{mg} / \mathrm{dL}$ ).
\end{abstract}

Conclusions: There was a non-linear association between plasma ApoE levels and the risk of EBB-induced bleeding. Higher plasma ApoE concentrations (> $5.9 \mathrm{mg} / \mathrm{dL}$ ) are the independent risk factor for hemorrhage during EBB in patients with lung cancer.

Keywords: Apolipoprotein E, Lung cancer, Endobronchial biopsy, Bleeding

\section{Background}

Patients with lung cancer are the main subjects who need bronchoscopy. Currently, bronchoscopic biopsy is indispensable in histopathological diagnosis of endobronchial exophytic lesions [1]. Common biopsy procedures include endobronchial forceps biopsy, cryobiopsy, bronchial brushing, transbronchial lung biopsy and transbronchial needle aspiration [2,3]. Endobronchial biopsy (EBB) has been used for the diagnosis of lung disease for over

\footnotetext{
* Correspondence: saibinwang@hotmail.com

${ }^{1}$ Department of Respiratory Medicine, Jinhua Municipal Central Hospital, No.

365, East Renmin Road, Jinhua, Zhejiang Province 321000, China

Full list of author information is available at the end of the article
}

40 years, and is still one of the most widely used biopsy modalities to date [4]. However, hemorrhage is a very common complication for bronchoscopists during EBB. Malignant lesions are more likely subjected to bleed upon biopsy than benign mucosal lesions [5]. The rate of EBB-induced hemorrhage is over $30.0 \%$ in patients with lung cancer [6], and massive biopsy-related hemorrhage could be life-threatening $[7,8]$.

Several risk factors have been proposed for hemorrhage during bronchoscopy, including mechanical ventilation, immunosuppression, thrombocytopenia, anti-coagulant or anti-platelet therapy, severe liver and kidney disease, heart failure, and pulmonary arterial hypertension [9-11].

(C) The Author(s). 2018 Open Access This article is distributed under the terms of the Creative Commons Attribution 4.0 International License (http://creativecommons.org/licenses/by/4.0/), which permits unrestricted use, distribution, and 
However, whether these proposed risk factors are in reality associated with hemorrhage during EBB, is still debatable $[12,13]$. In fact, most patients who undergo EBB do not deal with the aforementioned risk factors in clinical practice.

Apolipoprotein E (ApoE) plays a pivotal role in lipoprotein metabolism in the human body. During the last few decades, several studies have demonstrated that ApoE is associated with an increased risk of subarachnoid hemorrhage [14], hypertensive cerebral hemorrhage [15], amyloid angiopathy and angiorrhexis [16, 17]. The increased risk of hemorrhage in these disorders, at least in part, may be attributed to ApoE expression.

We hypothesized that plasma ApoE may be associated with the incidence of biopsy-induced bleeding events, and had the potential to be a biomarker for EBB-induced bleeding. The aim of this study was to explore the association of plasma ApoE with the risk of EBB-induced bleeding in patients with lung cancer.

\section{Methods}

\section{Patient population}

Retrospective review was done on medical records of consecutive patients who underwent EBB and were diagnosed with lung cancer at the Jinhua Municipal Central Hospital between January 2014 and February 2018. The collected patients met the following criteria: a. adult patients with endobronchial local exophytic lesions who underwent forceps biopsies; b. patients diagnosed with a primary lung cancer. Patients with the following "proposed risk factors" were excluded from the study, including bleeding tendencies, active bleeding, platelets $<50 \times 10^{3} / \mu \mathrm{l}$, continuous anti-coagulant or/and anti-platelet therapy, severe liver and kidney disease, heart failure, mechanical ventilation, pulmonary arterial hypertension, lung transplant, and immunosuppression.

Patients were classified into two groups: the bleeding group (those who received hemostasis maneuvers during $\mathrm{EBB}$ ) and the non-bleeding group (those who did not need hemostasis maneuvers, or did not experience hemorrhage during EBB).

The following details were collected: age, sex, systolic blood pressure (SBP), diastolic blood pressure (DBP), location of the lesions (central airways referred to the trachea, left main bronchi, right main bronchi, and right middle bronchus; whereas peripheral bronchi referred to the left and right lobar bronchi), histological types, TNM stage of cancer (stage I and II as an early stage, and stage III and IV as an advanced stage), and comorbidities (chronic obstructive pulmonary disease (COPD), coronary heart disease (CHD), hypertension, and diabetes). Blood tests included ApoE levels, total cholesterol (TC), triglyceride, low density lipoprotein cholesterol (LDLC), high density lipoprotein cholesterol (HDLC), C-reactive protein (CRP), white blood cell counts, neutrophils, hemoglobin, platelet counts, prothrombin time, activated partial thromboplastin time, aspartate aminotransferase (AST), and alanine aminotransferase (ALT). Blood tests were performed on the first visit or admission, three days prior to EBB.

This study was approved by the ethics committee of Jinhua Hospital of Zhejiang University (No. 2017101003). The requirement for informed consent was waived because the data were anonymous.

\section{Bronchoscopic procedures}

Bronchoscopic procedures were performed under general anesthesia. We used Propofol $(1.0 \mathrm{mg} / \mathrm{kg})$ for induction and (3.0-6.0 mg/kg/h) for anesthesia maintenance. Remifentanil $(5.0-10.0 \mu \mathrm{g} / \mathrm{kg} / \mathrm{h})$ was used for analgesia. Cisatracurium was used for the induction of neuromuscular blockade, as needed. A laryngeal mask airway (LMA) (Well Lead Medical Co., Ltd., Guangzhou, China) was used, and the bronchoscopic procedures were performed via LMA. Patients were ventilated using a closed circuit connected to the ventilator during the bronchoscopy. Fiberoptic bronchoscopy (BF-1 T60, Olympus Corp., Tokyo, Japan) was performed on all patients by two experienced bronchoscopists.

Generally, three to five biopsies were performed in each patient at the same location of the lesion using forceps biopsies [2], but only one biopsy was performed when some endobronchial lesions bled significantly following the first biopsy attempt. Cold water $\left(4{ }^{\circ} \mathrm{C}\right.$ physiological saline), diluted adrenalin (1:10000), and argon plasma coagulation (APC) were used for hemostasis.

\section{Statistical analysis}

Descriptive statistics were used to summarize baseline characteristics. Age was indicated as median (range), categorical variables were expressed as a number (percentage), and blood test values were presented as mean \pm standard error of mean. Unpaired t-tests, Pearson chi-squared tests or the Fisher's exact, were tested between two groups comparison, as appropriate. We performed multiple regression analysis to estimate the independent relationship between plasma ApoE concentrations and the risk of EBB-induced bleeding, with an adjustment for potential confounders. A two-piecewise linear regression was used to test the threshold effect of ApoE on EBB-induced bleeding using a smoothing function. All analyses were performed using $\mathrm{R}$ (The R Foundation; https://www.r-project.org) software and Empower (X\&Y solutions, Inc., Boston, MA; http:// www.empowerstats.com). A value of $P<0.05$ was considered statistically significant.

\section{Results}

Of 615 consecutive patients, 224 (36.4\%) hemorrhaged during $\mathrm{EBB}$, and they were subjected to hemostasis maneuvers (cold water, diluted adrenalin or/and APC). No 
Table 1 Baseline Characteristics and Blood tests of the Study Participants

\begin{tabular}{|c|c|c|}
\hline \multirow[t]{2}{*}{ Characteristics } & \multicolumn{2}{|c|}{ Biopsy-induced bleeding } \\
\hline & No $(n=391)$ & Yes $(n=224$ \\
\hline Age (y), median (range) & $65.00(32-85)$ & $65.50(36-82$ \\
\hline \multicolumn{3}{|l|}{ Sex, n (\%) } \\
\hline Female & $94(24.04)$ & $41(18.30)$ \\
\hline Man & $297(75.96)$ & $183(81.70)$ \\
\hline \multicolumn{3}{|l|}{ Smoking, n (\%) } \\
\hline Never & $158(40.41)$ & $68(30.36)$ \\
\hline Former & $56(14.32)$ & $44(19.64)$ \\
\hline Current & $177(45.27)$ & $112(50.00)$ \\
\hline $\mathrm{SBP}(\mathrm{mmHg})$ & $132.34 \pm 0.98$ & $130.01 \pm 1.37$ \\
\hline $\mathrm{DBP}(\mathrm{mmHg})$ & $77.95 \pm 0.58$ & $78.60 \pm 0.82$ \\
\hline \multicolumn{3}{|l|}{ Location of lesion, n (\%) } \\
\hline Peripheral bronchi & 362 (92.58) & $175(78.12)$ \\
\hline Central airway & $29(7.42)$ & $49(21.88)$ \\
\hline \multicolumn{3}{|l|}{ Stage, n (\%) } \\
\hline Early & $237(60.61)$ & $98(44.95)$ \\
\hline Advanced & 154 (39.39) & $120(55.05)$ \\
\hline \multicolumn{3}{|l|}{ Histological types, n (\%) } \\
\hline Adenocarcinoma & $130(33.25)$ & $36(16.07)$ \\
\hline Squamous cell carcinoma & $175(44.76)$ & $134(59.82)$ \\
\hline $\mathrm{SCLC}$ & $67(17.14)$ & $41(18.30)$ \\
\hline Others & $19(4.86)$ & $13(5.80)$ \\
\hline \multicolumn{3}{|l|}{ COPD, n (\%) } \\
\hline No & 365 (93.35) & $208(92.86)$ \\
\hline Yes & $26(6.65)$ & $16(7.14)$ \\
\hline \multicolumn{3}{|l|}{ Hypertension, n (\%) } \\
\hline No & $297(75.96)$ & $166(74.11)$ \\
\hline Yes & $94(24.04)$ & $58(25.89)$ \\
\hline \multicolumn{3}{|l|}{ Diabetes, n (\%) } \\
\hline No & 370 (94.63) & $214(95.54)$ \\
\hline Yes & $21(5.37)$ & $10(4.46)$ \\
\hline \multicolumn{3}{|l|}{ CHD, n (\%) } \\
\hline No & 379 (96.93) & $216(96.43)$ \\
\hline Yes & $12(3.07)$ & $8(3.57)$ \\
\hline Triglyceride (mmol/L) & $1.24 \pm 0.03$ & $1.15 \pm 0.05$ \\
\hline TC (mmol/L) & $4.19 \pm 0.06$ & $4.10 \pm 0.06$ \\
\hline HDLC (mmol/L) & $1.18 \pm 0.02$ & $1.12 \pm 0.02$ \\
\hline LDLC (mmol/L) & $2.84 \pm 0.04$ & $2.77 \pm 0.05$ \\
\hline Apo E (mg/dL) & $3.90 \pm 0.07$ & $3.65 \pm 0.10$ \\
\hline Apo B (g/L) & $1.02 \pm 0.02$ & $0.99 \pm 0.02$ \\
\hline Homocysteine $(\mu \mathrm{mol} / \mathrm{L})$ & $15.05 \pm 0.43$ & $20.48 \pm 5.23$ \\
\hline WBC $\left(\times 10^{9} / \mathrm{L}\right)$ & $7.31 \pm 0.16$ & $7.62 \pm 0.22$ \\
\hline Neutrophils (×109/L) & $5.23 \pm 0.16$ & $5.70 \pm 0.22$ \\
\hline
\end{tabular}

Table 1 Baseline Characteristics and Blood tests of the Study Participants (Continued)

\begin{tabular}{|c|c|c|}
\hline \multirow[t]{2}{*}{ Characteristics } & \multicolumn{2}{|c|}{ Biopsy-induced bleeding } \\
\hline & No $(n=391)$ & Yes $(n=224)$ \\
\hline Hemoglobin (g/L) & $127.66 \pm 1.69$ & $126.96 \pm 1.71$ \\
\hline platelets $\left(\times 10^{9} / \mathrm{L}\right)$ & $229.10 \pm 4.34$ & $241.23 \pm 6.63$ \\
\hline CRP (mg/L) & $21.81 \pm 1.73$ & $29.81 \pm 2.61$ \\
\hline PT (S) & $16.04 \pm 1.22$ & $13.90 \pm 0.74$ \\
\hline APTT (S) & $38.83 \pm 1.38$ & $35.58 \pm 1.15$ \\
\hline ALT (IU/L) & $22.27 \pm 0.91$ & $21.48 \pm 1.39$ \\
\hline AST (IU/L) & $26.54 \pm 0.73$ & $26.52 \pm 1.20$ \\
\hline \multicolumn{3}{|c|}{$\begin{array}{l}\text { SBP systolic blood pressure, } D B P \text { diastolic blood pressure, } S C L C \text { small-cell lung } \\
\text { carcinoma, COPD Chronic obstructive pulmonary disease, } C H D \text { coronary heart } \\
\text { disease, } T C \text { total cholesterol, } H D L C \text { high density lipoprotein cholesterol, } L D L C \\
\text { low density lipoprotein cholesterol, } A p O \text { apolipoprotein, WBC white blood cell, } \\
C R P C \text {-reactive protein, } P T \text { prothrombin time, APTT activated partial thromboplastin } \\
\text { time, } A L T \text { alanine aminotransferase, } A S T \text { aspartate aminotransferase }\end{array}$} \\
\hline
\end{tabular}

case of severe bleeding was recorded. Baseline characteristics and blood test results are presented in Table 1.

The plasma ApoE levels were higher in the non-bleeding group compared to those in the bleeding group (Table 2, $P<0.05)$. In addition, the location of the lesion, histological types, stage, smoking history, HDLC, and CRP level positively correlated with EBB-induced hemorrhage as assessed by univariate analysis (Table 2 ).

Table 3 shows the association between plasma ApoE and the risk of EBB-induced bleeding after adjusting for the location of the lesion, histological types, stage, smoking history, HDLC, and CRP level (adjust I), and on combined adjustment of risk factors judged by clinical significance (age, sex, diabetes, hypertension, COPD, CHD, SBP, DBP, TC, triglyceride, LDLC, ALT, and AST) (adjust II). We found that middle levels of ApoE (3.5-5.9 mg/dL) associated well with a decreased risk of EBB-induced bleeding when compared to lower levels $(<3.5 \mathrm{mg} / \mathrm{L})$ (odds ratio [OR], 0.64; 95\% confidence interval [CI], 0.43-0.94; $P<$ $0.05)$; however higher levels of $\mathrm{ApoE}(\geqq 6.0 \mathrm{mg} / \mathrm{L})$ did not associate with a decreased incidence of EBB-induced bleeding when compared to low level of ApoE $(<3.5 \mathrm{mg} / \mathrm{L})$ (OR, 0.85; 95\% CI, 0.41-1.76; $P>0.05$ ).

Further, we adjusted smoothed plots that suggested a nonlinear relationship between plasma ApoE and the risk of EBB-induced bleeding (Fig. 1). There were threshold effects between plasma ApoE levels and the risk of EBB-induced bleeding in two-piecewise linear regression analysis (Table 4). The risk of EBB-induced bleeding increased with increasing ApoE levels above the turning point (ApoE > $5.9 \mathrm{mg} / \mathrm{L}$ ) (Table 4; OR, 1.49; 95\% CI, 1.03-2.16; $P<0.05)$.

\section{Discussion}

To get a clearer understanding of the risks involved in hemorrhage during bronchoscopic biopsies, we studied 
Table 2 Univariate Analysis of Possible Influencing Factors of the Risk of Biopsy-induced Bleeding

\begin{tabular}{|c|c|c|}
\hline \multirow[t]{2}{*}{ Variables } & \multicolumn{2}{|c|}{ Biopsy-induced Bleeding } \\
\hline & $\overline{\mathrm{OR}}(95 \% \mathrm{Cl})$ & $P$ value \\
\hline Age (y), median (range) & $1.01(0.99,1.03)$ & 0.1909 \\
\hline \multicolumn{3}{|l|}{ Sex, n (\%) } \\
\hline Female & Ref. & \\
\hline Man & $1.41(0.94,2.13)$ & 0.0990 \\
\hline \multicolumn{3}{|l|}{ Smoking, n (\%) } \\
\hline Never & Ref. & \\
\hline Former & $1.83(1.12,2.97)$ & 0.0153 \\
\hline Current & $1.47(1.02,2.13)$ & 0.0411 \\
\hline $\mathrm{SBP}(\mathrm{mmHg})$ & $0.99(0.99,1.00)$ & 0.1595 \\
\hline $\mathrm{DBP}(\mathrm{mmHg})$ & $1.00(0.99,1.02)$ & 0.5100 \\
\hline \multicolumn{3}{|l|}{ Location of lesion, n (\%) } \\
\hline Peripheral bronchi & Ref. & \\
\hline Central airway & $3.50(2.13,5.72)$ & $<0.0001$ \\
\hline \multicolumn{3}{|l|}{ Stage, n (\%) } \\
\hline Early & Ref. & \\
\hline Advanced & $1.88(1.35,2.63)$ & 0.0002 \\
\hline \multicolumn{3}{|l|}{ Histological types, n (\%) } \\
\hline Adenocarcinoma & Ref. & \\
\hline Squamous cell carcinoma & $2.77(1.79,4.26)$ & $<0.0001$ \\
\hline $\mathrm{SCLC}$ & $2.21(1.29,3.78)$ & 0.0037 \\
\hline Others & $2.47(1.11,5.48)$ & 0.0260 \\
\hline \multicolumn{3}{|l|}{ COPD, n (\%) } \\
\hline No & Ref. & \\
\hline Yes & $1.08(0.57,2.06)$ & 0.8155 \\
\hline \multicolumn{3}{|l|}{ Hypertension, n (\%) } \\
\hline No & Ref. & \\
\hline Yes & $1.10(0.76,1.61)$ & 0.6085 \\
\hline \multicolumn{3}{|l|}{ Diabetes, n (\%) } \\
\hline No & Ref. & \\
\hline Yes & $0.82(0.38,1.78)$ & 0.6214 \\
\hline \multicolumn{3}{|l|}{ CHD, n (\%) } \\
\hline No & Ref. & \\
\hline Yes & $1.17(0.47,2.91)$ & 0.7356 \\
\hline Triglyceride (mmol/L) & $0.80(0.61,1.05)$ & 0.1084 \\
\hline $\mathrm{TC}(\mathrm{mmol} / \mathrm{L})$ & $0.91(0.77,1.07)$ & 0.2707 \\
\hline $\mathrm{HDLC}(\mathrm{mmol} / \mathrm{L})$ & $0.52(0.31,0.88)$ & 0.0146 \\
\hline LDLC (mmol/L) & $0.89(0.71,1.11)$ & 0.2856 \\
\hline Apo E (mg/dL) & $0.89(0.80,1.00)$ & 0.0488 \\
\hline Apo B $(g / L)$ & $0.76(0.46,1.27)$ & 0.2949 \\
\hline Homocysteine $(\mu \mathrm{mol} / \mathrm{L})$ & $1.00(0.99,1.01)$ & 0.4514 \\
\hline WBC (×109/L) & $1.03(0.98,1.08)$ & 0.2423 \\
\hline Neutrophils (×109/L) & $1.04(0.99,1.10)$ & 0.0856 \\
\hline
\end{tabular}

Table 2 Univariate Analysis of Possible Influencing Factors of the Risk of Biopsy-induced Bleeding (Continued)

\begin{tabular}{|c|c|c|}
\hline \multirow[t]{2}{*}{ Variables } & \multicolumn{2}{|c|}{ Biopsy-induced Bleeding } \\
\hline & $\mathrm{OR}(95 \% \mathrm{Cl})$ & $P$ value \\
\hline Hemoglobin (g/L) & $1.00(0.99,1.00)$ & 0.7850 \\
\hline platelets (× 109/L) & $1.00(1.00,1.00)$ & 0.1130 \\
\hline CRP (mg/L) & $1.01(1.00,1.01)$ & 0.0093 \\
\hline PT (S) & $0.99(0.98,1.01)$ & 0.2445 \\
\hline APTT (S) & $0.99(0.98,1.00)$ & 0.1430 \\
\hline $\mathrm{ALT}(\mathrm{IU} / \mathrm{L})$ & $1.00(0.99,1.01)$ & 0.6193 \\
\hline AST (IU/L) & $1.00(0.99,1.01)$ & 0.9870 \\
\hline
\end{tabular}

EBB-induced bleeding in patients with lung cancer. The present study showed that plasma ApoE was associated with EBB-induced bleeding in a non-linear pattern. In order to reduce the risk of biopsy-induced bleeding, especially for massive bleeding during bronchoscopy, it may be appropriate to maintain the levels of plasma ApoE between $3.5 \mathrm{mg} / \mathrm{dL}$ and $5.9 \mathrm{mg} / \mathrm{dL}$ prior to aggressive biopsy on endobronchial exophytic lesions in lung cancer patients.

Hemorrhage is a very common complication during bronchoscopy, especially when biopsies are performed. It has been noted that malignant lesions are more prone to bleed upon biopsy than benign mucosal lesions [5], and massive blood loss may occur more frequently following EBB [18]. Several risk factors have been proposed to be associated with hemorrhage during bronchoscopy, including immunosuppression, mechanical ventilation, uncontrolled hypertension, severe pulmonary arterial hypertension, thrombocytopenia, anti-coagulant and anti-platelet use, lung transplant, heart failure, liver and kidney disease, and bleeding tendencies [9-11]. However, most of these proposed risk factors are conflicting and lack supporting evidence $[12,13]$. To the best of our knowledge, there is no effective indicator available to date for predicting the risk of hemorrhage during bronchoscopy.

ApoE is traditionally an important modulator of many stages of lipoprotein metabolism in the human body. There have also been reports in recent years that ApoE may play a role in some hemorrhagic diseases, such as hypertensive cerebral hemorrhage and subarachnoid hemorrhage $[14,15]$. It has been demonstrated that ApoE is associated with an increased risk of amyloid angiopathy and angiorrhexis [16, 17]. It affects the formation of abnormal vessels and pericytes in neurological disorders [14]. Additionally, ApoE impacts the immunomodulatory and oxidative status, which may be 
Table 3 Multivariate Regression Analysis of ApoE With the Risk of Biopsy-induced Bleeding

\begin{tabular}{|c|c|c|c|}
\hline \multirow[t]{2}{*}{ ApoE (mg/dL) } & \multicolumn{3}{|c|}{ Biopsy-induced bleeding OR (95\% Cl) P-value } \\
\hline & Non-adjust & Adjust $\left.\right|^{a}$ & Adjust $\|^{\mathrm{b}}$ \\
\hline$<3.5$ & Ref. & Ref. & Ref. \\
\hline $3.6-5.9$ & $0.59(0.42,0.84) 0.0029$ & $0.63(0.43,0.91) 0.0140$ & $0.64(0.43,0.94) 0.0228$ \\
\hline$\geqq 6.0$ & $0.74(0.39,1.39) 0.3487$ & $0.86(0.44,1.71) 0.6731$ & $0.85(0.41,1.76) 0.6634$ \\
\hline
\end{tabular}

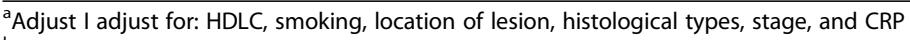

${ }^{b}$ Adjust II adjust for: Sex, Age, SBP, DBP, smoking, TC, triglyceride, HDLC, LDLC, location of lesion, histological types, stage, diabetes, hypertension, COPD, CHD, ALT, AST, and CRP

Apo apolipoprotein, HDLC high density lipoprotein cholesterol, CRP C-reactive protein, SBP systolic blood pressure, DBP diastolic blood pressure, $T C$ total cholesterol, $L D L C$ low density lipoprotein cholesterol, COPD Chronic obstructive pulmonary disease, CHD coronary heart disease, $A L T$ alanine aminotransferase, $A S T$ aspartate aminotransferase

associated with poor neurological outcomes after traumatic brain injury and hemorrhage $[19,20]$.

Our study found that plasma ApoE levels associated well with the incidence of EBB-induced bleeding after adjustment for the main confounding risk factors (HDLC, smoking, location of lesion, histological types, stage, and CRP), or on combined adjustment for factors considered to be clinically relevant (sex, age, SBP, DBP, TC, triglyceride, LDLC, diabetes, hypertension, COPD, CHD, ALT, and AST); the strength of this association did not change. There was a non-linear association between plasma ApoE and the risk of EBB-induced bleeding. We further revealed a piecewise effect of ApoE concentrations. Between $3.5-5.9 \mathrm{mg} / \mathrm{dL}$, ApoE was associated with lower risk of EBB-induced bleeding, whereas higher $(>5.9 \mathrm{mg} / \mathrm{dL}$ ) concentrations of ApoE were associated with an increased risk

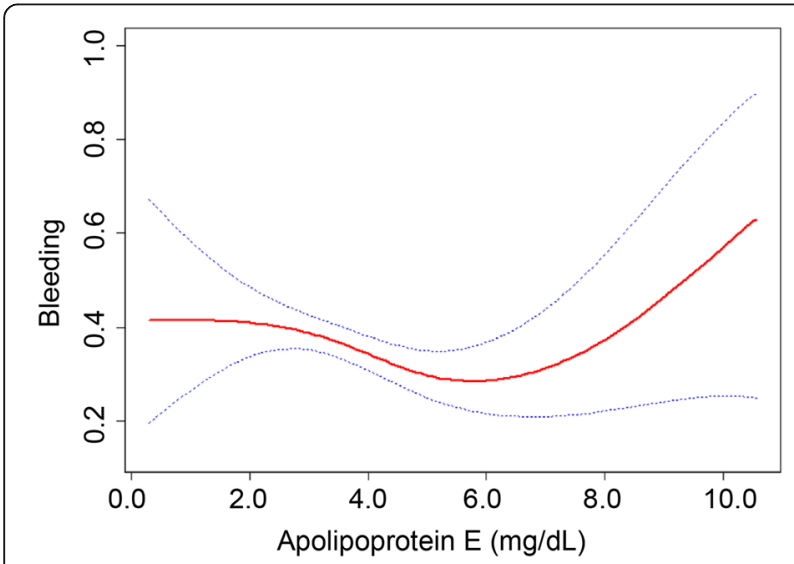

Fig. 1 The smooth curve fitting showed the association between the risk of EBB-induced bleeding and plasma ApoE levels after adjusting the relative confounding factors (HDLC, smoking, location of lesion, histological types, stage, and (RP), and factors considered to be clinically relevant, which include sex, age, SBP, DBP, TC, triglyceride, LDLC, diabetes, hypertension, COPD, CHD, ALT, and AST. Dotted lines represented the upper and lower 95\% confidence intervals. $\mathrm{EBB}=$ endobronchial biopsy; $\mathrm{ApoE}=$ apolipoprotein $\mathrm{E} ; \mathrm{HDLC}=$ high density lipoprotein cholesterol; $\mathrm{CRP}=\mathrm{C}$-reactive protein; $\mathrm{SBP}=$ systolic blood pressure; $\mathrm{DBP}=$ diastolic blood pressure; $\mathrm{TC}=$ total cholesterol; LDLC = low density lipoprotein cholesterol; $C O P D=$ Chronic obstructive pulmonary disease; $\mathrm{CHD}=$ coronary heart disease; $\mathrm{ALT}=$ alanine aminotransferase; $\mathrm{AST}=$ aspartate aminotransferase of EBB-induced bleeding. Although the mechanism underlying the concentration-dependent relationship between ApoE and the risk of EBB-induced bleeding remains to be elucidated, this finding may be valuable information for the selection and preoperative preparation of biopsy patients.

The present study is the first to reveal the relationship between plasma ApoE and the risk of biopsy-induced bleeding during bronchoscopy. Strengths of this study are, the inclusion of consecutive patients, having fixed bronchoscopists, using only one biopsy maneuver, and a relatively fixed number of biopsies, as well as a relatively fixed ApoE detection time before bronchoscopy. However, some limitations of the current study are worth noting. Firstly, it is still challenging to accurately measure bleeding during bronchoscopy [21]. We could not provide a quantitative measurement of the volume of biopsy-induced bleeding in our study. Patients were divided into a bleeding group or a non-bleeding group based only on whether they received hemostasis during EBB. Therefore, this classification may not accurately group patients with minimal bleeding. Secondly, it is well known that ApoE has three isoforms in humans, namely ApoE2, ApoE3 and ApoE4 [20]. We however did

Table 4 Threshold effect analysis of ApoE on EBB-induced bleeding using two-piecewise linear regression

\begin{tabular}{|c|c|c|}
\hline \multirow[t]{2}{*}{ Inflection point of ApoE (mg/dL) } & \multicolumn{2}{|c|}{ Biopsy-induced Bleeding ${ }^{a}$} \\
\hline & OR $(95 \% \mathrm{Cl})$ & $P$ value \\
\hline \multicolumn{3}{|l|}{ Inflection point I } \\
\hline$<3.5$ & $1.00(0.70,1.4$ & \\
\hline$>3.5$ & $0.67(0.47,0.9$ & \\
\hline \multicolumn{3}{|l|}{ Inflection point II } \\
\hline$<5.9$ & $0.82(0.69,0.9$ & \\
\hline$>5.9$ & $1.49(1.03,2.1$ & \\
\hline
\end{tabular}

adjust for: Sex, Age, SBP, DBP, smoking, TC, triglyceride, HDLC, LDLC, location of lesion, histological types, stage, diabetes, hypertension, COPD, CHD, ALT, AST, and CRP

$A p o$ apolipoprotein, HDLC high density lipoprotein cholesterol, CRP C-reactive protein, SBP systolic blood pressure, DBP diastolic blood pressure, $T C$ total cholesterol, LDLC low density lipoprotein cholesterol, COPD Chronic obstructive pulmonary disease, $C H D$ coronary heart disease, $A L T$ alanine aminotransferase, AST aspartate aminotransferase 
not do any further genotyping of ApoE because information on these variables was unavailable in the retrospective data. Therefore, the results of this study may only be representative of Asian lung cancer population in East China.

\section{Conclusions}

In conclusion, our study showed that plasma ApoE is associated with the risk of EBB-induced bleeding in patients with lung cancer in a non-linear pattern, and the relative safe levels of ApoE may be 3.5-5.9 mg/dL. Our findings highlight the importance of ApoE levels in bleeding during EBB and may have implications for risk assessment and risk modification prior to bronchoscopy. However, future prospective studies involving a larger number of subjects and basic research are warranted to fully evaluate its clinical value and the mechanisms involved.

\section{Abbreviations \\ ALT: Alanine aminotransferase; APC: Argon plasma coagulation; ApoE: Apolipoprotein E; APTT: Activated partial thromboplastin time; AST: Aspartate aminotransferase; CHD: Coronary heart disease; CI: Confidence interval; COPD: Chronic obstructive pulmonary disease; CRP: C-Reactive protein; DBP: Diastolic blood pressure; EBB: Endobronchial biopsy; HDLC: High density lipoprotein cholesterol; LDLC: Low density lipoprotein cholesterol; LMA: Laryngeal mask airway; OR: Odds ratio; PT: Prothrombin time; SBP: Systolic blood pressure; TC: Total cholesterol; WBC: White blood cell}

\section{Acknowledgements}

We appreciate the help and support of all the participants involved in the study.

\section{Funding}

This study was supported by the Medical and Health Science and Technology Plan Project of Zhejiang Province (No. 2018263197 to S. W.), and the Youth Research Fund of Jinhua Hospital of Zhejiang University (No. JY2017205 to S. W.).

\section{Availability of data and materials}

The datasets used and/or analysed during the current study are available from the corresponding author on reasonable request.

\section{Authors' contributions}

SW contributed substantially to the study design, data analysis and interpretation, the writing of the manuscript, and takes responsibility for the integrity of the data and the accuracy of the data analysis. QY and XL contributed to data collection, data analysis, data interpretation, and manuscript revision. All authors read and approved the final manuscript.

\section{Ethics approval and consent to participate}

This study was approved by the ethics committee of Jinhua Hospital of Zhejiang University (No. 2017101003). The requirement for informed consent was waived because the data were anonymous.

\section{Consent for publication}

Not applicable.

\section{Competing interests}

The authors declare that they have no competing interests.

\section{Publisher's Note}

Springer Nature remains neutral with regard to jurisdictional claims in published maps and institutional affiliations.

\section{Author details}

'Department of Respiratory Medicine, Jinhua Municipal Central Hospital, No. 365, East Renmin Road, Jinhua, Zhejiang Province 321000, China. ${ }^{2}$ Department of Medical Records Quality Management, Jinhua Municipal Central Hospital, No. 365, East Renmin Road, Jinhua, Zhejiang Province 321000, China. ${ }^{3}$ Department of Laboratory Medicine, Jinhua Municipal Central Hospital, No. 365, East Renmin Road, Jinhua, Zhejiang Province 321000, China.

Received: 11 May 2018 Accepted: 10 July 2018

Published online: 21 July 2018

\section{References}

1. Rivera MP, Mehta AC, Wahidi MM. Establishing the diagnosis of lung cancer: diagnosis and management of lung cancer, 3rd ed: American College of Chest Physicians evidence-based clinical practice guidelines. Chest. 2013;143:e142S-65S.

2. Rivera MP, Detterbeck F, Mehta AC. Diagnosis of lung cancer: the guidelines. Chest. 2003;123:129S-36S.

3. Aktas Z, Gunay E, Hoca NT, Yilmaz A, Demirag F, Gunay S, Sipit T, Kurt EB. Endobronchial cryobiopsy or forceps biopsy for lung cancer diagnosis. Annals of Thoracic Medicine. 2010:5:242-6.

4. Fallon J, Medford ARL. Endobronchial and transbronchial biopsy experience: a United Kingdom survey. Thorac Cancer. 2017;8:291-5.

5. Ozgül MA, Turna A, Yildiz P, Ertan E, Kahraman S, Yilmaz V. Risk factors and recurrence patterns in 203 patients with hemoptysis. Tuberk Toraks. 2006;54:243-8.

6. Wang $\mathrm{S}, \mathrm{Ye} \mathrm{Q}, \mathrm{Tu}$ J, Song Y. The location, histological type and stage of lung cancer are associated with bleeding during endobronchial biopsy. Cancer Manag Res. 2018;10:1251-7.

7. Herth FJF. Bronchoscopy and bleeding risk. Eur Respir Rev. 2017;26:170052.

8. Chinsky K. Bleeding risk and bronchoscopy: in search of the evidence in evidence-based medicine. Chest. 2005;127:1875-7.

9. Herth FJ, Becker HD, Ern A. Aspirin does not increase bleeding complications after transbronchial biopsy. Chest. 2002;122:1461-4.

10. Diette GB, Wiener CM, White $P$ Jr. The higher risk of bleeding in lung transplant recipients from bronchoscopy is independent of traditional bleeding risks: results of a prospective cohort study. Chest. 1999;115:397-402.

11. Brickey DA, Lawlor DP. Transbronchial biopsy in the presence of profound elevation of the international normalized ratio. Chest. 1999;115:1667-71.

12. Carr IM, Koegelenberg CF, von Groote-Bidlingmaier F, Mowlana A, Silos K, Haverman T, Diacona AH, Bolligeret CT. Blood loss during flexible bronchoscopy: a prospective observational study. Respiration. 2012;84:312-8.

13. Zahreddine I, Atassi K, Fuhrman C. Impact of prior biological assessment of coagulation on the hemorrhagic risk of fiberoptic bronchoscopy. Rev Mal Respir. 2003;20:341-6.

14. Jofre-Monseny L, Minihane AM, Rimbach G. Impact of apoE genotype on oxidative stress, inflammation and disease risk. Mol Nutr Food Res. 2008:52:131-45.

15. Misra UK, Kalita J, Somarajan BI. Recurrent intracerebral hemorrhage in patients with hypertension is associated with APOE gene polymorphism: a preliminary study. J Stroke Cerebrovasc Dis. 2013;22:758-63.

16. Tzourio C, Arima H, Harrap S, Anderson C, Godin O, Woodward M, Neal B, Bousser MG, Chalmers J, Cambien F, et al. APOE genotype, ethnicity, and the risk of cerebral hemorrhage. Neurology. 2008;70:1322-8.

17. Greenberg SM, Vonsattel JP, Segal AZ, Chiu RI, Clatworthy AE, Liao A, Hyman BT, Rebeck GW. Association of apolipoprotein E epsilon2 and vasculopathy in cerebral amyloid angiopathy. Neurology. 1998;50:961-5.

18. Jin F, Mu D, Chu D, Fu E, Xie Y, Liu T. Severe complications of bronchoscopy. Respiration. 2008; 76:429-33.

19. Friedman G, Froom P, Sazbon L, Grinblatt I, Shochina M, Tsenter J, Babaey S, Yehuda B, Groswasser Z. Apolipoprotein E-epsilon4 genotype predicts a poor outcome in survivors of traumatic brain injury. Neurology. 1999;52:244-8.

20. Bell RD, Winkler EA, Singh I, Sagare AP, Deane R, Wu Z, Holtzman DM, Betsholtz C, Armulik A, Sallstrom J, et al. Apolipoprotein E controls cerebrovascular integrity via cyclophilin a. Nature. 2012;485:512-6.

21. Schumann C, Hetzel M, Babiak AJ, Hetzel J, Merk T, Wibmer T, Lepper PM, Krüger S. Endobronchial tumor debulking with a flexible cryoprobe for immediate treatment of malignant stenosis. J Thorac Cardiovasc Surg. 2010;139:997-1000 